\title{
The Accuracy of the Surgical Peritoneal Cancer Index in Patients with Peritoneal Metastases of Colorectal Cancer
}

\author{
Nadine L. de Boer ${ }^{a} \quad$ Alexandra R.M. Brandt-Kerkhof ${ }^{a}$ Eva V.E. Madsen ${ }^{a}$ \\ Michael Doukas $^{\mathrm{b}}$ Cornelis Verhoef $^{\mathrm{a}}$ Jacobus W.A. Burger ${ }^{\mathrm{a}, \mathrm{c}}$ \\ aDepartment of Surgical Oncology, Erasmus MC Cancer Institute, Rotterdam, The Netherlands; \\ bDepartment of Pathology, Erasmus MC, Rotterdam, The Netherlands; 'Department of Surgery, \\ Catharina Cancer Institute, Eindhoven, The Netherlands
}

\section{Keywords}

Peritoneal cancer index · Peritoneal metastases $\cdot$ Colorectal cancer · Cytoreductive surgery and hyperthermic intraperitoneal chemotherapy

\begin{abstract}
Introduction: The peritoneal cancer index $(\mathrm{PCl})$ is one of the most important prognostic factors in patients with peritoneal metastases from colorectal cancer undergoing cytoreductive surgery and hyperthermic intraperitoneal chemotherapy (CRS-HIPEC). The PCl is determined during laparotomy by 2 experienced surgeons and plays a major role in the decision to proceed with CRS-HIPEC. The primary objective of this study was to determine the accuracy of the surgical $\mathrm{PCl}(\mathrm{sPCl}$ ) by comparing it with the $\mathrm{PCl}$ confirmed by the pathologist ( $\mathrm{pPCl}$ ). Methods: All consecutive patients who underwent CRS-HIPEC for colorectal peritoneal metastases between February 2015 and June 2018 were identified. Relevant patient- and tumor-related characteristics were collected. Results: In total, 119 patients were included, 60 males (50.4\%). The median age was 64 (IQR 55-71). The median $\mathrm{sPCl}(\mathrm{sPCl}=11$, IQR 6-16) was significantly higher than the median $\mathrm{pPCl}(\mathrm{pPCl}=8$, IQR 3-13, $p<0.001)$. The total
\end{abstract}

karger@karger.com www.karger.com/dsu

Karger $\stackrel{\text { ' }}{5}$

GOPEN ACCESS
(C) 2021 The Author(s)

Published by S. Karger AG, Basel

This is an Open Access article licensed under the Creative Commons Attribution-NonCommercial-4.0 International License (CC BY-NC) (http://www.karger.com/Services/OpenAccessLicense), applicable to the online version of the article only. Usage and distribution for commercial purposes requires written permission.
$\mathrm{pPCl}$ was lower than the total $\mathrm{sPCl}$ in 80 patients $(67.2 \%)$. In 21 patients $(17.6 \%)$, the $\mathrm{sPCl}$ was overestimated with $\geq 5$ points. Small lesions are more likely to be negative. In patients that underwent resection of their primary tumor prior to CRS-HIPEC, the difference between the $\mathrm{SPCl}$ and $\mathrm{pPCl}$ was significantly larger $(p<0.05)$. Conclusions: Surgical calculation of the $\mathrm{PCl}$ often results in overestimation. Far-reaching consequences are tied to the macroscopic evaluation of the $\mathrm{sPCl}$, but this evaluation seems not very reliable.

(c) 2021 The Author(s)

Published by S. Karger AG, Basel

\section{Introduction}

Currently, cytoreductive surgery and hyperthermic intraperitoneal chemotherapy (CRS-HIPEC) is the most aggressive treatment option for selected patients with peritoneal disseminated colorectal cancer [1-4]. The extent of disease, scored by the peritoneal cancer index (PCI), and the completeness of cytoreduction are the 2 main prognostic factors for the outcomes of these patients after CRS-HIPEC [3-6]. The PCI is scored during CRS-HIPEC and is based on the macroscopic evaluation by the surgeon. The total PCI score can range from 0 to 
Fig. 1. Peritoneal cancer index (reprinted from Gilly et al. [8], Copyright 2006, with permission from Elsevier).

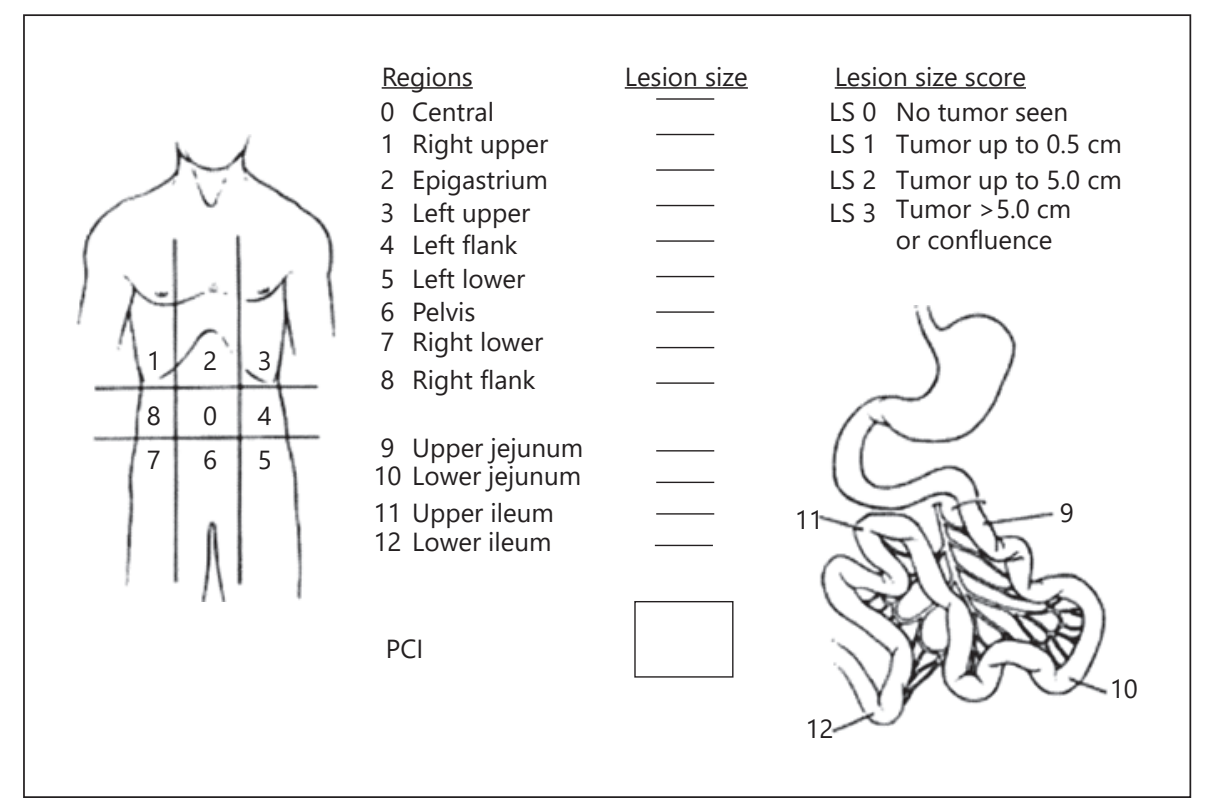

39. Frozen sections may also be used to determine whether a peritoneal lesion is malignant $[3,5,7,8]$.

Within the last 2 decades, many have tried to set a cutoff beyond which CRS-HIPEC should be contraindicated, without much consensus [4]. In the Netherlands, CRS-HIPEC is not considered beneficial in case of a PCI of $>20[9,10]$. The decision to "do" or to "do not" proceed with CRS-HIPEC has major consequences for patients. When a patient is not eligible for CRS-HIPEC, the prognosis is dismal (3-15 months) and the only remaining therapy is palliative systemic therapy or best supportive care [11-13].

Important treatment decisions are made based on the PCI scored during surgery. Little is known about how reliable this macroscopic evaluation actually is. The aim of this current study is to determine the accuracy of the surgical PCI (sPCI) by comparing it with the PCI confirmed by the pathologist (pPCI).

\section{Materials and Methods}

Objectives

The primary objective of this study is to determine the accuracy of the sPCI by comparing it with the pPCI. Secondary objectives are to investigate different factors that might influence the accuracy of the sPCI, that is, lesion size, prior resection of the primary tumor, and neoadjuvant chemotherapy, and to identify specific PCI regions in which the PCI is frequently under- or overestimated. An additional objective is to investigate the prognostic value of the sPCI.

\section{Patients}

This retrospective study was conducted in the Erasmus MC Cancer Institute in Rotterdam, The Netherlands. The Erasmus MC Cancer Institute started performing CRS-HIPEC in 2014. All consecutive patients and relevant patient- and tumor-related characteristics were collected in a prospectively maintained database. This database was used to identify the patients included in this study.

Inclusion criteria for this study were all patients that underwent CRS-HIPEC for peritoneal disseminated colorectal cancer. All patients were operated between February 2015 and June 2018. General contraindications for CRS-HIPEC are $>3$ liver metastases and/ or extra-abdominal metastases. If no PCI score was mentioned in the surgical report or the pathological report, patients were excluded. There were no age restrictions.

\section{Preoperative Screening}

All patients had standardized preoperative evaluation to ensure suitability for CRS-HIPEC $[6,7,14,15]$. In most patients, a diagnostic laparoscopy was performed prior to CRS-HIPEC to estimate the PCI. If during laparoscopy the sPCI was $\leq 20$, patients were planned for an elective CRS-HIPEC procedure.

\section{Cytoreductive Surgery and Hyperthermic Intraperitoneal Chemotherapy}

All Dutch centers performing CRS-HIPEC have to adhere to standards set by the governing body SONCOS. These standards state that CRS-HIPEC centers have to perform at least 20 CRSHIPEC per year. Centers that initiated a CRS-HIPEC program required approval of the Dutch Peritoneal Oncology Group (DPOG) and received standardized DPOG training and proctoring $[14,15]$. The implementation of this standardized national treatment protocol resulted in good overall survival rates and, although experience of CRS-HIPEC centers differed, survival and surgical outcomes between Dutch hospitals are similar $[14,15]$. 
After training and proctoring, the Erasmus MC Cancer Institute started performing CRS-HIPEC in 2014 with a team of 3 surgeons specialized in gastrointestinal surgery. Before the inclusion period of this current study, 30 operations were performed of which 26 CRS-HIPEC procedures and 4 open-close procedures were due to irresectable disease.

All procedures included in this current study were performed by 2 of the 3 specialized surgeons together. After midline laparotomy, a thorough assessment of the extent of disease was conducted and the sPCI was determined. The PCI is calculated by giving each of the 13 abdominal regions $(0-12)$ a score ranging from 0 (no tumor seen) to 3 (tumor $>5 \mathrm{~cm}$ or confluence) (Fig. 1). The total PCI is calculated by adding all 13 regions and can thus range from 0 to $39[7,8]$.

If complete cytoreduction was considered feasible, primary tumor (if still present), involved abdominal organs, involved parietal surfaces, and all peritoneal implants were resected and labeled per PCI region for pathological evaluation. The greater omentum was resected routinely in all patients. All suspected tissues were sent to the pathologist for confirmation of the malignant diagnosis. Administration of HIPEC was by the open colosseum technique, and regimens used were mitomycin $\mathrm{C}\left(35 \mathrm{mg} / \mathrm{m}^{2}\right)$ or oxaliplatin $(460$ $\left.\mathrm{mg} / \mathrm{m}^{2}\right)$ in combination with systemic folinic acid $\left(20 \mathrm{mg} / \mathrm{m}^{2}\right)$ and 5 -fluorouracil $\left(400 \mathrm{mg} / \mathrm{m}^{2}\right)$ for a 90 - or 30 -min perfusion period, respectively. After the perfusion, intestinal bowel anastomoses were created if necessary. Completeness of cytoreduction was scored after CRS-HIPEC using Sugarbaker's completeness of cytoreduction score: $\mathrm{CC}-0$ (no visible peritoneal carcinomatosis after CRS), CC-1 (nodules persisting $<2.5 \mathrm{~mm}$ after CRS), CC-2 (nodules persisting between $2.5 \mathrm{~mm}$ and $2.5 \mathrm{~cm}$ ), and CC-3 (nodules persisting $>2.5 \mathrm{~cm}$ ) [5].

\section{Histopathological Evaluation}

The resected tissues from the affected regions were separately labeled and sent to the pathologist. The pathologist involved in this study is a dedicated gastrointestinal tract pathologist with specific expertise in the field of peritoneal metastases (PM). All tissues were assessed macroscopically (visual inspection and palpation) for nodular lesions. These lesions were described and measured. If the size of the lesion was not specifically described in the pathology report, the lesion size reported in the operative report was maintained. After fixation in formalin, representative sections were taken. Followed by paraffin embedding, sections of $5 \mu \mathrm{m}$ were prepared and stained with hematoxylin and eosin (H\&E). All H\&E slides were examined microscopically. Macroscopic as well as microscopic findings were described in the pathology reports. The pPCI was calculated in retrospect by comparing the size and diagnosis of the lesions in the pathology reports with the corresponding $\mathrm{PPCI}$ mentioned per region in the operative reports.

\section{Statistical Analysis}

Continuous variables were presented as median with interquartile range (IQR). Categorical variables were presented as absolute numbers and percentages. Medians were compared using the Mann-Whitney U test. Two-sided $p$ values $<0.05$ were considered statistically significant.

The Kaplan-Meier method was used for survival analysis. Overall survival (OS) was calculated from the date of surgery until death or last follow-up. Patients were censored when alive at last follow-up date. All patients were divided into 4 PCI categories (0-

The Accuracy of the Surgical Peritoneal Cancer Index
Table 1. Baseline characteristics $(N=119)$

\begin{tabular}{|c|c|c|}
\hline & $N$ & $\%$ \\
\hline Age at operation & 64 & {$[55-71]^{*}$} \\
\hline \multicolumn{3}{|l|}{ Gender } \\
\hline Male & 60 & 50.4 \\
\hline Female & 59 & 49.6 \\
\hline \multicolumn{3}{|l|}{ Location of primary tumor } \\
\hline Right colon & 52 & 43.7 \\
\hline Transverse colon & 7 & 5.9 \\
\hline Left colon & 12 & 10.1 \\
\hline Sigmoid & 32 & 26.9 \\
\hline Rectum & 16 & 13.4 \\
\hline \multicolumn{3}{|l|}{ Metastases } \\
\hline Synchronous & 51 & 42.9 \\
\hline Metachronous & 68 & 57.1 \\
\hline \multicolumn{3}{|l|}{ Primary tumor } \\
\hline Resected during prior surgery & 98 & 82.4 \\
\hline In situ at time of CRS-HIPEC & 21 & 17.6 \\
\hline \multicolumn{3}{|l|}{ Neoadjuvant treatment with CTx } \\
\hline Yes & 10 & 8.4 \\
\hline No & 109 & 91.6 \\
\hline \multicolumn{3}{|l|}{ Complete cytoreduction score } \\
\hline CC- 0 & 119 & 100 \\
\hline$\geq \mathrm{CC}-1$ & - & - \\
\hline \multicolumn{3}{|l|}{ Type of chemotherapy } \\
\hline MMC & 102 & 85.7 \\
\hline Oxali/5FU/LV & 17 & 14.3 \\
\hline Procedure time, min & 375 & {$[311-437]^{*}$} \\
\hline Blood loss, mL & 1,050 & {$[600-1,600]^{*}$} \\
\hline ICU stay, days & 3 & {$[2-3]^{*}$} \\
\hline Hospital admission, days & 16 & {$[12-22]^{*}$} \\
\hline Overall survival, months & 36 & {$[18-\mathrm{NR}]^{*}$} \\
\hline Follow-up, months & 23 & {$[13-31]^{*}$} \\
\hline
\end{tabular}

CRS-HIPEC, cytoreductive surgery and hyperthermic intraperitoneal chemotherapy; CTx, chemotherapy; MMC, mitomycin C; Oxali/5FU/LV, oxaliplatin, 5-fluorouracil, leucovorin; ICU intensive care unit. * Median and interquartile range.

$5,6-10,11-15$, and $\geq 16$ ) based on their sPCI and pPCI. We calculated the median and 1-, 2-, and 3-year survival for each group. Statistical analyses were performed using Statistical Package for Social Sciences (SPSS), version 24.0.0 for Windows (IBM Corporation, Armonk, NY, USA).

\section{Results}

\section{Patient Cohort}

Between February 2015 and June 2018, 157 patients with PM of colorectal origin were planned for CRSHIPEC. In 23 patients, CRS-HIPEC was aborted (openclose procedure) because of a high sPCI or unresectable (liver) metastases. A total of 134 patients underwent CRS- 


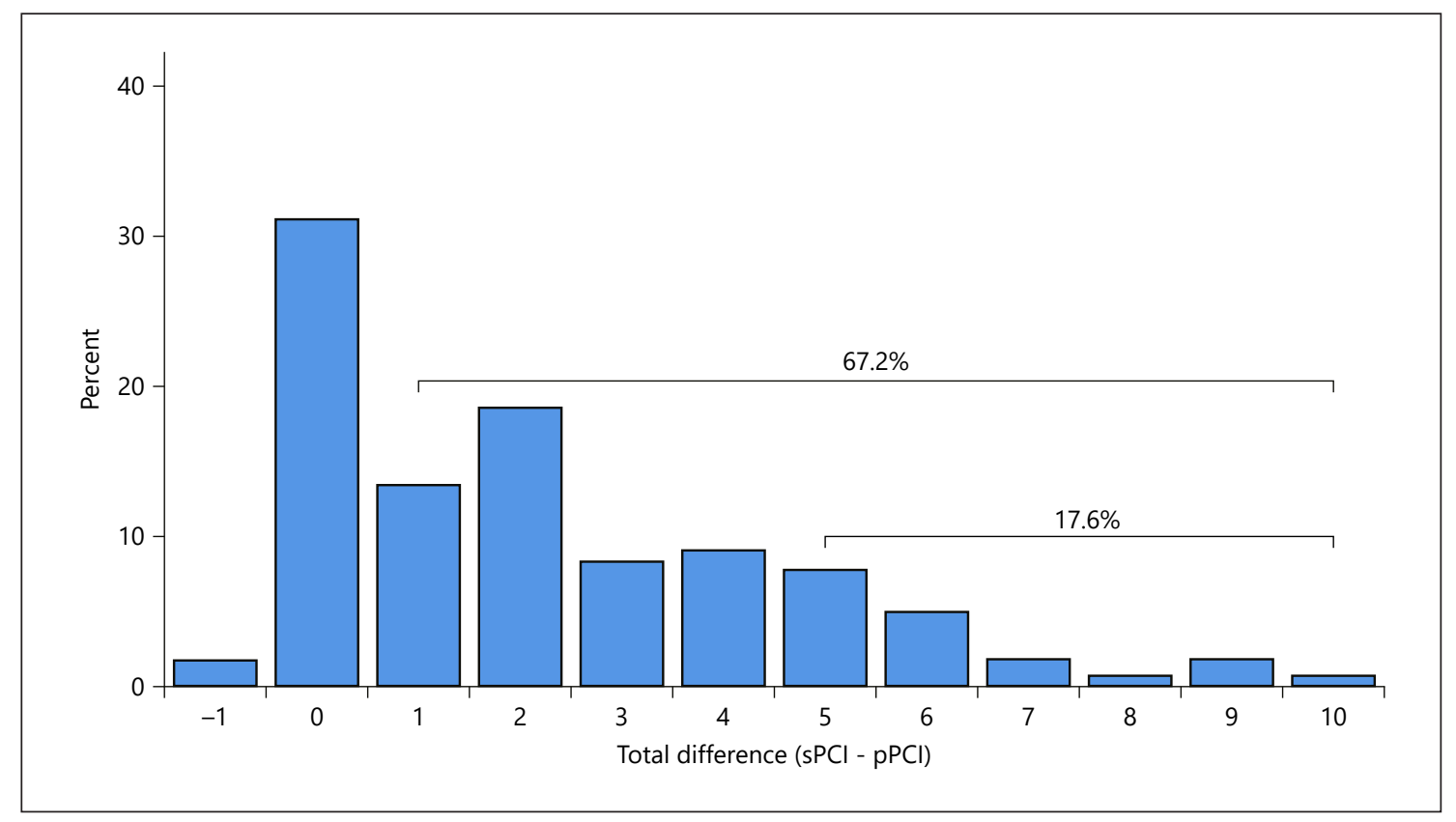

Fig. 2. Difference in total sPCI score versus total pPCI $(N=119)$. sPCI, surgical peritoneal cancer index; pPCI, pathological peritoneal cancer index.

HIPEC. In 15 of those 134 patients (11.2\%), the pathologist did not report his/her findings regarding the separate PCI regions and therefore these patients were excluded. In total, 119 patients were included in this study. All 119 patients underwent complete cytoreduction (CC-0). Baseline characteristics are described in Table 1.

\section{Peritoneal Cancer Index}

The median sPCI scored by the surgeons during laparotomy was 11 (IQR 6-16). The median $\mathrm{pPCI}$ was significantly lower ( $\mathrm{pPCI}=8$, IQR $3-13, p<0.001)$. The median difference between the sPCI and pPCI was 2 (IQR 0-4). We compared the sPCI with the pPCI for all 13 PCI regions separately. All but one (region 2) had a significantly lower pPCI $(p<0.05)$. The total pPCI was lower than the sPCI in $80 / 119$ patients (67.2\%). In 21 patients (17.6\%), the total sPCI was overestimated with $\geq 5$ points (Fig. 2 ).

\section{Lesion Size}

Small lesions are more likely to be negative. Region score 1 was given by the surgeon 273 times, of which 104 (38.1\%) were negative during pathological assessment. Region score 2 was given 359 times and negative in 79 (22.0\%). Region score 3 was given the least, 97 times, but only 4 (4.1\%) were not malignant during pathological assessment. Adjustment in lesion size was rare and oc- curred 6 times; in 3 cases, the region score went up 1 point, and 3 times the region score was 1 point lower.

\section{Resection of Primary Tumor}

In this study, 51 patients with synchronous PM and 68 with metachronous PM were included. Of the patients with synchronous metastases, 31 (60.8\%) underwent prior resection of the primary tumor, and in 20 (39.2\%), the primary tumor was still in situ. In all patients with metachronous PM, except one, the primary tumor was resected $(n=67,98.5 \%)$.

In patients where the primary tumor was resected before CRS-HIPEC, the median difference between the sPCI and pPCI was 2 (IQR 0-4). This median difference was significantly lower in patients that still had their primary tumor in situ at the time of CRS-HIPEC (0; IQR $0-2, p=0.021)$.

\section{Neoadjuvant Chemotherapy}

Only 10 patients $(8.4 \%)$ included in this study received neoadjuvant chemotherapy. The median difference between the sPCI and PPCI in patients treated with neoadjuvant chemotherapy was 1.5 (IQR 0-4). The median difference of patients not treated with neoadjuvant chemotherapy was 2 (IQR $0-4$ ). This difference was not significant $(p=0.957)$. 
Table 2. Difference in survival

\begin{tabular}{|c|c|c|c|c|c|c|c|c|c|c|}
\hline $\begin{array}{l}\text { PCI } \\
\text { categories }\end{array}$ & \multicolumn{5}{|l|}{ sPCI } & \multicolumn{5}{|l|}{ pPCI } \\
\hline $0-5$ & $25(21.2)$ & NR [33.0-NR] & 87.5 & 82.0 & 61.5 & $42(35.6)$ & NR [33.0-NR] & 89.8 & 86.6 & 55.7 \\
\hline $6-10$ & $44(37.3)$ & $36.0[36.0-\mathrm{NR}]$ & 93.7 & 83.8 & 37.7 & $33(28.0)$ & $\mathrm{NR}[25.0-\mathrm{NR}]$ & 90.8 & 80.3 & 64.2 \\
\hline
\end{tabular}

PCI, peritoneal cancer index; sPCI, surgical peritoneal cancer index; pPCI, pathological peritoneal cancer index; IQR, interquartile range; NR, not reached.

\section{Prognostic Value}

The median survival was calculated for each of the 4 PCI groups, low PCI to high PCI (Table 2). About twothirds $(n=80,67.2 \%)$ stayed in the same PCI group after the pPCI was determined by the pathologist. However, 39 patients $(32.8 \%)$ moved $\geq 1$ category down. No patients moved to a higher group. Of those 39 patients, $15(12.6 \%)$ moved from $6-10$ to $0-5,12$ (10.1\%) from $11-15$ to $6-10$, and $8(6.7 \%)$ from $\geq 16$ to $11-15$. Four $(3.4 \%)$ patients moved down 2 pPCI categories; 2 moved from 11-15 to $0-5$, and another 2 from $\geq 16$ to $6-10$.

\section{Discussion}

This study suggests that overestimation of the sPCI is common $(67.2 \%)$, irrespective of the location of the lesions. In $17.6 \%$, the overestimation was $\geq 5$ points. We conclude that far-reaching consequences (open-close procedure) are tied to the macroscopic evaluation of the sPCI by the surgeon, but that this evaluation seems not very reliable.

We tried to identify specific regions in which the pPCI often turned out lower than the sPCI. However, when we compared each sPCI region score separately with the corresponding pPCI, all but one were significantly lower. Small lesions were more likely to be negative during pathological assessment. Down- or upstaging in lesion size was rare. This means that surgeons are good in determining the size of lesions during surgery, but the distinction between malignant or nonmalignant lesions is more challenging.

The most plausible explanation for overestimation of the sPCI is that the majority of the patients $(82.4 \%)$ underwent surgery before CRS-HIPEC. We showed that in patients that underwent resection of their primary tumor before undergoing CRS-HIPEC, the difference between the sPCI and pPCI was significantly larger. Prior surgery could have resulted in intra-abdominal abnormalities, such as adhesions, calcifications, or fibrosis. Differentiation between reactive changes or tumor can be difficult, even for experienced surgeons. In the future, intraoperative fluorescence imaging might be able to provide guidance in the differentiation between benign or malignant lesions $[16,17]$.

Other studies on this subject have suggested that negative pathological findings could be a result of the response on neoadjuvant chemotherapy $[18,19]$. This study could not confirm this. In the Netherlands, upfront CRSHIPEC is standard of care. Therefore, only $8.4 \%$ of the patients included received neoadjuvant chemotherapy. There was no difference between the sPCI and pPCI in the patients treated and not treated with neoadjuvant chemotherapy.

Another explanation could be sampling error. During CRS-HIPEC, large resections are performed, and after macroscopic evaluation of all the tissues, the pathologist examines the most suspect nodules from each region microscopically, since examination of all the tissue microscopically is simply not feasible.

Although the main goal of this study was determination of the accuracy of the sPCI, determination of the pPCI after CRS-HIPEC may also have consequences for patients. In this study, 32.8\% moved to a lower PCI category and $3.4 \%$ even moved down 2 PCI categories after calculation of the PPCI. Therefore, we believe that determination of the pPCI postoperative could be of added value and it could possibly help in the decision-making around the administration of adjuvant treatment. 
This study has limitations. First is the retrospective nature of the study. Second is that the pathologist only received tissues that the surgeon deemed suspect. Therefore, in most cases, the pathologist was only able to downgrade the sPCI. In 9 cases, a region was initially scored 0 , but a suspicious lesion was found, resected, and during pathological analysis diagnosed as malignant. In one patient, this resulted in a total pPCI which was higher than the sPCI. Another limitation is the risk of interobserver variability. All CRS-HIPEC procedures were performed by 2 specialized surgeons; the sPCI was determined by consensus between the 2 surgeons performing the procedure.

With data on the pPCI from patients in whom an open-close procedure was performed because of a PCI of $>20$, we could have determined whether the sPCI actually results in unwarranted open-close procedures. However, since removal of tissue in patients undergoing open-close procedures holds no benefit for the patient, it was not considered ethical. Our findings do however suggest that liberal use of perioperative frozen sections is warranted to confirm the sPCI before terminating CRS and HIPEC procedures. In some countries, a sPCI of $>16$ is considered a contraindication for CRS-HIPEC $[4,9,10]$. In this study, the number of patients with a sPCI of $\geq 16$ decreased with $1 / 3$ rd (from 30 to 20 ) after determination of the pPCI.

In our center, 23 open-close procedures were performed during the inclusion period, of which 4/23 were performed because of unresectable liver metastases. In $19 / 23$, the sPCI was too high, and $8 / 19$ had a sPCI between 21 and 25 . We cannot conclude how many of these patients would have had a PPCI of $\leq 20$, because, as mentioned above, patients in whom an open-close procedure was performed, no or minimal biopsies were taken. However, it is likely that some of these patients would have had a $\mathrm{pPCI}$ of $\leq 20$.
Although the pPCI is probably more accurate, the sPCI is used and will continue to be used for decisionmaking to "do" or "do not" continue with CRS-HIPEC. Therefore, we believe that if peritoneal disease seems resectable during CRS-HIPEC but the PCI is deemed too high to continue, confirmation of the diagnosis with intraoperative frozen sections is recommended. In some patients, open-close procedures will be prevented. Further research is warranted to determine whether the pPCI is a better scoring method/more precise prognostic factor for oncological outcomes after CRS-HIPEC.

\section{Statement of Ethics}

This retrospective study was approved by the local medical ethics committee of the Erasmus MC (Registration No. MEC-20181286), and in this specific study, the ethical committee approved the lack of informed consent from the patients.

\section{Conflict of Interest Statement}

The authors have no conflicts of interest to declare.

\section{Funding Sources}

No funding was received for this study.

\section{Author Contributions}

N.L.d.B. drafted this manuscript and performed the data analysis. C.V., J.W.A.B., and A.R.M.B. initiated this study. A.R.M.B., E.V.E.M., and J.W.A.B. performed CRS-HIPEC surgery on the patients included in this study. M.D. is the pathologist involved in this study. All authors read and revised the manuscript. All authors approved the final manuscript.

\section{References}

1 Sugarbaker PH. Peritonectomy procedures. Ann Surg. 1995;221(1):29-42.

2 Sugarbaker PH. Cytoreductive surgery plus hyperthermic perioperative chemotherapy for selected patients with peritoneal metastases from colorectal cancer: a new standard of care or an experimental approach? Gastroenterol Res Pract. 2012;2012:309417.

3 Sugarbaker PH, Jablonski KA. Prognostic features of 51 colorectal and 130 appendiceal cancer patients with peritoneal carcinomatosis treated by cytoreductive surgery and intraperitoneal chemotherapy. Ann Surg. 1995; 221(2):124-32.
4 Klaver CE, Groenen H, Morton DG, Laurberg S, Bemelman WA, Tanis PJ, et al. Recommendations and consensus on the treatment of peritoneal metastases of colorectal origin: a systematic review of national and international guidelines. Colorectal Dis. 2017;19(3): 224-36.

5 Harmon RL, Sugarbaker PH. Prognostic indicators in peritoneal carcinomatosis from gastrointestinal cancer. Int Semin Surg Oncol. 2005;2(1):3.
6 Esquivel J, Sticca R, Sugarbaker P, Levine E, Yan TD, Alexander R, et al. Cytoreductive surgery and hyperthermic intraperitoneal chemotherapy in the management of peritoneal surface malignancies of colonic origin: a consensus statement. Society of Surgical Oncology. Ann Surg Oncol. 2007;14(1):128-33.

7 Jacquet P, Sugarbaker PH. Clinical research methodologies in diagnosis and staging of patients with peritoneal carcinomatosis. Cancer Treat Res. 1996;82:359-74. 
8 Gilly FN, Cotte E, Brigand C, Monneuse O, Beaujard AC, Freyer G, et al. Quantitative prognostic indices in peritoneal carcinomatosis. Eur J Surg Oncol. 2006;32(6):597-601.

9 Goere D, Souadka A, Faron M, Cloutier AS, Viana B, Honore C, et al. Extent of colorectal peritoneal carcinomatosis: attempt to define a threshold above which HIPEC does not offer survival benefit: a comparative study. Ann Surg Oncol. 2015;22(9):2958-64.

10 Faron M, Macovei R, Goéré D, Honoré C, Benhaim L, Elias D. Linear relationship of peritoneal cancer index and survival in patients with peritoneal metastases from colorectal cancer. Ann Surg Oncol. 2016; 23(1):114-9.

11 Hompes D, Boot $\mathrm{H}$, van Tinteren $\mathrm{H}$, Verwaal $\mathrm{V}$. Unresectable peritoneal carcinomatosis from colorectal cancer: a single center experience. J Surg Oncol. 2011;104(3):269-73.
12 Klaver YL, Simkens LH, Lemmens VE, Koopman $\mathrm{M}$, Teerenstra S, Bleichrodt RP, et al. Outcomes of colorectal cancer patients with peritoneal carcinomatosis treated with chemotherapy with and without targeted therapy. Eur J Surg Oncol. 2012;38(7):617-23.

13 Sadeghi B, Arvieux C, Glehen O, Beaujard AC, Rivoire M, Baulieux J, et al. Peritoneal carcinomatosis from non-gynecologic malignancies: results of the EVOCAPE 1 multicentric prospective study. Cancer. 2000;88(2): 358-63.

14 Kuijpers AM, Mirck B, Aalbers AG, Nienhuijs SW, de Hingh IH, Wiezer MJ, et al. Cytoreduction and HIPEC in the Netherlands: nationwide long-term outcome following the Dutch protocol. Ann Surg Oncol. 2013; 20(13):4224-30.

15 Kuijpers AM, Aalbers AG, Nienhuijs SW, de Hingh IH, Wiezer MJ, van Ramshorst B, et al. Implementation of a standardized HIPEC protocol improves outcome for peritoneal malignancy. World J Surg. 2015;39(2):453-60.
16 Hentzen JEKR, de Jongh SJ, Hemmer PHJ, van der Plas WY, van Dam GM, Kruijff S. Molecular fluorescence-guided surgery of peritoneal carcinomatosis of colorectal origin: a narrative review. J Surg Oncol. 2018;118(2): 332-43.

17 de Boer E, Harlaar NJ, Taruttis A, Nagengast WB, Rosenthal EL, Ntziachristos V, et al. Optical innovations in surgery. Br J Surg. 2015; 102(2):e56-72.

18 Berger Y, Jacoby H, Kaufmann MI, Ben-Yaacov A, Westreich G, Sharon I, et al. Correlation between intraoperative and pathological findings for patients undergoing cytoreductive surgery and hyperthermic intraperitoneal chemotherapy. Ann Surg Oncol. 2019;26(4): 1103-9.

19 Passot G, You B, Boschetti G, Fontaine J, Isaac S, Decullier E, et al. Pathological response to neoadjuvant chemotherapy: a new prognosis tool for the curative management of peritoneal colorectal carcinomatosis. Ann Surg Oncol. 2014;21(8):2608-14. 